\title{
Factores asociados a la automedicación en adultos
}

\section{Factors associated with self-medication in adults}

\author{
Clara Esperanza Suqui Belesaca ${ }^{1}$, Rocio Parra Pérez*1, Stephany Paladines Calle ${ }^{1}$, Edison Gustavo Moyano \\ Brito $^{1}$ \\ ${ }^{1}$ Universidad Católica de Cuenca, Ecuador \\ *cparrap@ucacue.edu.ec
}

DOI: https://doi.org/10.26871/killkanasalud.v4i4.744

\begin{abstract}
Resumen
Introducción: En los países de Latinoamérica la automedicación es muy frecuente, es así que en países como México existe una prevalencia del $59 \%$ de esta problemática, en Chile un $72 \%$, en Argentina un $83 \%$, en Perú un $44 \%$ y en Ecuador un 57,8\%, a diferencia de los países como España que su prevalencia es de 5-10\%, esto puede deberse a que existe mayor concientización en esta población. Objetivo: Determinar los factores asociados a la automedicación en adultos que acuden al Centro de Salud Jima, periodo septiembre 2017 - febrero 2018. Materiales y Métodos: Se realizó un estudio descriptivo, transversal, de enfoque cuantitativo, en el cual el tamaño de la muestra fue de 169 personas. Los datos se obtuvieron mediante una encuesta y los mismos fueron procesados en el programa IBM SPSS STATISTICS versión 24, donde se realizó estadística descriptiva para cada una de las variables y la prueba Chi cuadrado de Pearson para medir la asociación entre ellas. Resultados: Se aplicó el cuestionario a 169 pacientes que acudieron al centro de salud Jima. Obteniendo como resultado que el 79,3\% de los participantes se han automedicado en alguna etapa de su vida, los fármacos más utilizados fueron los antigripales en un 46,6\% de la población, asimismo el 33,58\% de los pacientes reportaron sentirse influenciados por el personal de las farmacias para automedicarse. Conclusiones: Finalmente se logró observar que más de la mitad de los participantes reportaron automedicarse, por lo que es primordial realizar campañas informativas acerca de los efectos adversos de esta problemática y así evitar futuras complicaciones de las diversas patologías o incluso resistencias antibióticas.
\end{abstract}

Palabras clave: automedicación, autoadministración, errores de medicación, medicamentos sin prescripción, prescripción inadecuada.

\begin{abstract}
Introduction: In Latin American countries, self-medication is very frequent, so in countries like Mexico there is a prevalence of 59\% of this problem, in Chile 72\%, in Argentina 83\%, in Peru 44\%, in Ecuador 57,8\%, unlike countries like Spain that its prevalence is $5-10 \%$, this may be due to the greater awareness of this population. Objective: To determine the factors associated with self-medication in patients who attend the Jima Health Center, period September 2017 - February 2018. Materials and Methods: A descriptive, cross-sectional study with a quantitative approach was carried out, in which the size of the sample was 169 people. The data were obtained by means of a survey and they were processed in the IBM SPSS STATISTICS version 24 program, where descriptive statistics were performed for each of the variables and the Pearson Chi square test to measure the association between them. Results: The questionnaire was applied to 169 patients who attended the Jima health center. Obtaining as a result that $79.3 \%$ of the participants have self-medicated at some stage of their life, the most used drugs were anti-flu in $46.6 \%$ of the population, also $33.58 \%$ of the patients reported feeling influenced by pharmacy staff to self-medicate. Conclusions: Finally, it was observed that more than half of the participants reported self-medicating, so it is essential to carry out information campaigns about the adverse effects of this problem and thus avoid future complications of the various pathologies or even antibiotic resistance.
\end{abstract}

Keywords: self-medication, self-administration, medication errors, non-prescription medications, inadequate prescription.

\section{Introducción}

Se define a la automedicación como el consumo de fármacos sin la prescripción de un médico. ${ }^{1,2}$ La Organización Mundial de la Salud dio a conocer que el $50 \%$ de los fármacos se distribuyen y se prescriben de forma inapropiada, así también la mitad de los pacientes no toman la medicación de manera correcta. ${ }^{3}$

Esta conducta genera un problema a nivel mundial, de acuerdo a un estudio realizado en Latinoamérica, solo el $34 \%$ de los medicamentos dispensados fueron prescritos; 
observando que un alto índice de medicamentos fueron vendidos sin prescripción médica. ${ }^{4}$

Se conocen varios factores que fomentan a la automedicación, en la actualidad la publicidad influye en gran medida para que aumente la prevalencia de este problema, asimismo se ha observado que las personas siguen recomendaciones de familiares, amigos o la comunidad en general. ${ }^{4,5}$

Uno de los principales factores que fomenta esta problemática; es la condición del sistema de salud de cada uno de los países, la restricción a la red de salud pública juega un papel fundamental, incluso el constante crecimiento del consumo de medicamentos se debe a la oferta y demanda de fármacos; las personas se sienten confundidas y sin una adecuada información. ${ }^{6-8}$

Es importante cuantificar la automedicación en nuestro país, ya que puede ocasionar distintos problemas en la salud de quien se automedica, prolongando o agravando una enfermedad; incluso puede causar resistencia antibiótica. Por tal motivo el objetivo del presente trabajo fue determinar la prevalencia y relacionar los factores que se asocian a la automedicación.

\section{Marco teórico}

\subsection{Antecedentes}

En Europa la prevalencia de la automedicación varía entre el 5 al $10 \%$, es una problemática que tiene una prevalencia muy inferior a los países de Latinoamérica, esto puede deberse al hecho de que son países desarrollados, que tienen normas y leyes de regulación diferentes. ${ }^{1,9}$

En Latinoamérica; la prevalencia de automedicación varia de un país a otro, en México es del $59 \%$, en Chile del $72 \%$, en Argentina del $83 \%$ y en Perú del 95,1\%. Encontrándose que la automedicación en estos países es muy frecuente, ya que al conocer la patología y saber de la farmacología de los medicamentos, tienden a realizar esta práctica por decisión propia o por consultas informales entre familiares, amistades o por influencia de algún medio de comunicación, evitando en lo posible realizar una visita médica, con la respectiva prescripción farmacológica. ${ }^{10}$

En un estudio realizado en Perú, se determinó que el $44 \%$ de la población se automedica; entre los factores relacionados los autores describen; el ingreso económico familiar y el nivel de instrucción. Siendo la cefalea y la gripe uno de los principales síntomas que se presentan para que una persona se automedique. ${ }^{11}$

En Ecuador, en un estudio realizado por Sánchez Francisco y colabores en la ciudad de Quito, se registró que la prevalencia de la automedicación en la población analizada fue del 57,8\%. ${ }^{12}$ Asimismo, en una investigación realizada en la ciudad de Cuenca, se detalla que la automedicación tiene un índice elevado, debido a la facilidad de conseguir medicamentos y por el acceso a una información sesgada, esto refleja el riesgo al que está expuesta la juventud, señalando que la prevalencia de la automedicación en el grupo analizado fue del $51 \%$; los medicamentos de mayor uso fueron los analgésicos y los antiinflamatorios y que las recomendaciones para el consumo de algún fármaco fue de un amigo o un familiar. ${ }^{13}$

\subsection{Medicamentos de venta libre en Ecuador}

El Ministerio de Salud Pública y la Agencia Nacional de Regulación, Control y Vigilancia Sanitaria, elaboraron un listado de medicamentos como antigripales, analgésicos, antiinflamatorios de venta libre. ${ }^{14,15}$

Tabla 1: Medicamentos de venta libre en Ecuador

\begin{tabular}{|c|c|}
\hline Nombre & $\begin{array}{l}\text { Tipo de Fárma- } \\
\text { co }\end{array}$ \\
\hline Acrogrip jarabe & Antigripal \\
\hline Antigripina plus & Antigripal \\
\hline Coldflu polvo antigripal & Antigripal \\
\hline Dristan triple action $\mathrm{NF}$ & Antigripal \\
\hline Finagrip día y noche tabletas & Antigripal \\
\hline Finalin gripe fenilefrina & Antigripal \\
\hline Fluzetrin f cápsulas & Antigripal \\
\hline Genfargrip tabletas & Antigripal \\
\hline Lemonflu miel & Antigripal \\
\hline Limonada caliente tapsin noche & Antigripal \\
\hline Nyflu cápsulas & Antigripal \\
\hline Singripal sobre & Antigripal \\
\hline Dextrometorfano jarabe $15 \mathrm{mg} / 5 \mathrm{ml}$ & Antitusígeno \\
\hline Limonada caliente tapsin día & Antitusígeno \\
\hline Metorfan forte jarabe & Antitusígeno \\
\hline Robitussin honey cough & Antitusígeno \\
\hline Theraflu dx adultos & Antitusígeno \\
\hline Sinutab plus ns & Antitusígeno \\
\hline Tapsin analgésico nocturno maver & Antitusígeno \\
\hline Teragrip día 500mg 30mg tabletas & Antitusígeno \\
\hline Ácido acetil salicílico $100 \mathrm{mg}$ tabletas & Analgésico \\
\hline Alka - seltzer doble acción & Analgésico \\
\hline Asawin $500 \mathrm{mg}$. Masticables & Analgésico \\
\hline Aspirina 500mg tabletas & Analgésico \\
\hline Aspirina advanced tabletas & Analgésico \\
\hline Donfiest lua granulado efervescente & Analgésico \\
\hline Dolorine $500 \mathrm{mg}$ tabletas & Analgésico \\
\hline Mejoral tabletas & Analgésico \\
\hline Paracetamol tabletas & Analgésico \\
\hline Termofin plus & Analgésico \\
\hline Aspirina direkt tabletas masticables & Antiinflamatorio \\
\hline
\end{tabular}

Fuente: Los autores 


\subsection{Factores asociados a la automedicación}

\subsubsection{Factores económicos}

Dentro de los factores económicos que provocan que las personas se automediquen, se encuentra el nivel socioeconómico bajo, debido a que algunas de las veces los individuos no cuentan con un empleo estable o con un sueldo que cubre las necesidades de salud familiar, por lo que no cuentan con el dinero para acudir a una consulta médica y les resulta más fácil automedicarse para resolver sus dolencias. ${ }^{16,17}$

\subsubsection{Factores sociales}

Los factores sociales que influyen en la automedicación se relaciona con la población que no asiste al médico, y deciden pedir consejos a sus familiares, amigos o al personal que atiende en farmacias o boticas; así también cuando presentan síntomas similares a experiencias patológicas anteriores, tienen la creencia de que la misma terapéutica farmacológica resolverá el problema. ${ }^{18}$

\subsubsection{Factores culturales}

Entendiendo como factores culturales a las tradiciones, estilos de vida, costumbres ancestrales, conocimientos artísticos y científicos. Además la publicidad de diversa información disponible en diferentes medios de comunicación, por parte de las empresas farmacéuticas, confunde a los individuos e influye a que la población consuma dichos fármacos. ${ }^{19}$

\subsection{Riesgos de la automedicación}

\subsubsection{Retraso del diagnóstico}

Esta problemática se presenta cuando existen algunos síntomas o dolencias por las que el individuo ingiere algún tipo de fármaco, enmascarando la sintomatología propia de la enfermedad provocando un retraso en el diagnóstico.

\subsubsection{Diagnóstico incorrecto}

La automedicación provoca que no se brinde el tratamiento adecuado de acuerdo al diagnóstico médico, ya que va a influir en la sintomatología y la dificultad del médico para dar un diagnóstico correcto. ${ }^{20}$

\section{Metodología}

Se realizó un estudio descriptivo, transversal, de enfoque cuantitativo, sobre una muestra de 169 pacientes mayores de 18 años que asistieron al Centro de Salud Jima. Para la recolección de la información acerca de los factores asociados a la automedicación, se utilizó una encuesta previamente diseñada para el estudio, y el control de sesgos de este instrumento se ajustó con base a una prueba piloto realizada a 30 personas del grupo de estudio.

Previamente a la ejecución de la encuesta se solicitó el consentimiento informado de cada uno de los participantes, además de ello la información recopilada fue utilizada de manera confidencial, respetando lo establecido en los derechos de Helsinki.

Una vez aplicada la encuesta, los datos fueron procesados en el programa estadístico IBM SPSS STATISTICS versión 24, en el cual se empleó estadística descriptiva para cada una de las variables de estudio y la prueba Chi cuadrado de Pearson para medir el grado de asociación entre ellas.

\section{Resultados}

De los 169 participantes encuestados, el 73,9\% correspondió al sexo femenino y el $26,1 \%$ al sexo masculino. De los cuales el 79,3\% asintió haberse automedicado en algún momento de su vida y el $20,7 \%$ restante no.

Asimismo, se evidencio que el 33,6\% de los participantes que se automedican señalaron sentirse influenciado por las recomendaciones del personal que labora en las farmacias, el $28,4 \%$ por criterio propio, el $26,1 \%$ por recomendaciones de familiares y el $11,9 \%$ por recomendaciones de amigos.

Con respecto al tipo de fármaco más usado para la automedicación, se constató que el 46,3\% de los pacientes consumen antigripales, el $35 \%$ AINES, el 10,3\% antibióticos y con una menor incidencia los antiespasmódicos con un $8,2 \%$.

En cuanto a la asociación entre la automedicación y los ingresos económicos, se constató que el 64,5\% de los participantes reciben ingresos económicos mensuales entre 386 a 500 dólares, de los cuales el 87,2\% reporto automedicarse. Además de ello, la prueba Chi cuadrado, demostró una asociación significativa entre las variables con un valor p de 0,002 . (Tabla 2)

Tabla 2: Asociación entre la automedicación y los ingresos económicos

\begin{tabular}{clcccccccc}
\hline & & \multicolumn{3}{c}{ Se automedica } & \multicolumn{3}{c}{ Total } & P \\
& & & Si & & & No & & & \\
& & $\mathrm{n}$ & $\%$ & $\mathrm{n}$ & $\%$ & $\mathrm{n}$ & $\%$ & \\
\hline \multirow{2}{*}{ Ingresos económicos } & $500-100$ & 26 & 81,3 & 6 & 18,7 & 32 & 18,9 & \\
& $386-500$ & 95 & 87,2 & 14 & 12,8 & 109 & 64,5 & 0.002 \\
& Inferior a 386 & 13 & 46,4 & 15 & 53,6 & 28 & 16,6 & \\
\hline
\end{tabular}

Fuente: Los autores 
Por otro lado, con respecto a la asociación entre el tipo de fármaco que más se usan para la automedicación y los medios de publicidad, se corroboró que el 51,6\% de los participantes han observado publicidades de antigripales en medios televisivos, siendo este medio el que más influye en la decisión de medicarse sin una prescripción médica. Observando de igual manera asociación significativa entre las variables, con un valor p de 0,003 . (Tabla 3 )

Tabla 3: Asociación entre los fármacos ingeridos y los medios de publicidad

\begin{tabular}{|c|c|c|c|c|c|c|c|c|c|c|c|c|c|c|}
\hline & & \multicolumn{10}{|c|}{ Medios de publicidad } & \multirow{2}{*}{\multicolumn{2}{|c|}{ Total }} & \multirow{3}{*}{$\mathrm{P}$} \\
\hline & & \multicolumn{2}{|c|}{ Televisión } & \multicolumn{2}{|c|}{ Periódicos } & \multicolumn{2}{|c|}{ Redes sociales } & \multicolumn{2}{|c|}{ Revistas } & \multicolumn{2}{|c|}{ Otros } & & & \\
\hline & & $\mathrm{n}$ & $\%$ & $\mathrm{n}$ & $\%$ & $\mathrm{n}$ & $\%$ & $\mathrm{n}$ & $\%$ & $\mathrm{n}$ & $\%$ & $\mathrm{n}$ & $\%$ & \\
\hline \multirow{5}{*}{ Fármacos } & Aines & 20 & 42,6 & 5 & 10,6 & 17 & 36,2 & 1 & 2,1 & 4 & 8,5 & 47 & 35,1 & \multirow{5}{*}{0.003} \\
\hline & Antigripales & 32 & 51,6 & 10 & 16,1 & 10 & 16,1 & 6 & 9,7 & 4 & 6,5 & 62 & 46,3 & \\
\hline & Antibióticos & 7 & 50 & 1 & 7,1 & 4 & 28,7 & 1 & 7,1 & 1 & 7,1 & 14 & 10,4 & \\
\hline & Antiespasmódicos & 4 & 36,4 & 1 & 9 & 6 & 54,6 & 0 & 0 & 0 & 0 & 11 & 8,2 & \\
\hline & Total & 63 & 47 & 17 & 12,7 & 37 & 27,6 & 8 & 6 & 9 & 6,7 & 134 & 100 & \\
\hline
\end{tabular}

Fuente: Los autores

Adicional a ello, en lo que se refiere a la asociación entre la sintomatología que presentan los participantes y quien les recomienda automedicarse, se evidencio que los individuos que presentan malestar general, en un $37,5 \%$ lo hacen por criterio propio, en un $30 \%$ por que se ven influenciados por el personal de farmacia, en un 22,5\% por recomendación de familiares y en un $10 \%$ por recomendación de amigos. Constatando de igual manera que las variables presentan asociación significativa, con un valor p de 0,002. (Tabla 4)

Tabla 4: Asociación entre los fármacos ingeridos y los medios de publicidad

\begin{tabular}{|c|c|c|c|c|c|c|c|c|c|c|c|c|}
\hline & & \multicolumn{2}{|c|}{$\begin{array}{l}\text { Por criterio } \\
\text { de ud mismo }\end{array}$} & \multicolumn{2}{|c|}{ Familiares } & \multicolumn{2}{|c|}{ Amigas/os } & \multicolumn{2}{|c|}{$\begin{array}{l}\text { Personal } \\
\text { farmacia }\end{array}$} & \multicolumn{2}{|c|}{ total } & \multirow[t]{2}{*}{$\mathrm{P}$} \\
\hline & & $\mathrm{n}$ & $\%$ & $\mathrm{n}$ & $\%$ & $\mathrm{n}$ & $\%$ & $\mathrm{n}$ & $\%$ & $\mathrm{n}$ & $\%$ & \\
\hline \multirow{4}{*}{$\begin{array}{l}\text { Ud. toma } \\
\text { medicación } \\
\text { cuando presenta } \\
\text { algún síntoma }\end{array}$} & Tos & 3 & 27,3 & 3 & 27,3 & 2 & 18,2 & 5 & 45,5 & 11 & 8,2 & \multirow{5}{*}{0.002} \\
\hline & Dolor Abdominal & 3 & 21,4 & 4 & 28,6 & 1 & 7,1 & 6 & 42,9 & 14 & 10,4 & \\
\hline & Malestar General & 15 & 37,5 & 9 & 22,5 & 4 & 10 & 12 & 30 & 40 & 29,9 & \\
\hline & Ninguno & 6 & 17,6 & 12 & 35,3 & 5 & 14,7 & 11 & 32,4 & 34 & 25,4 & \\
\hline \multicolumn{2}{|c|}{ Total } & 38 & 28,4 & 35 & 26,1 & 16 & 11,9 & 45 & 33,6 & 134 & 100 & \\
\hline
\end{tabular}

Fuente: Los autores

\section{Conclusiones y recomendaciones}

Finalmente, se logró observar que más de la mitad de los participantes se han automedicado en algún momento de su vida. De los cuales el mayor porcentaje refirió automedicarse por recomendaciones del personal que labora en las farmacias, asimismo reportaron que los antigripales son el tipo de fármaco que consumen con mayor frecuencia sin una prescripción médica.

Estos valores son alarmantes por lo que es importante educar a la población sobre el peligro que conlleva la automedicación mediante charlas educativas o ferias de salud. La educación se debe iniciar en los centros de salud para crear conciencia a la población ante dicha problemática. Adicional a ello, toda la publicidad de medicamentos de venta libre deberían tener mayor control y contar con la aprobación del Ministerio de Salud Pública.

\section{Fuente de Financiamiento}

Este estudio es autofinanciado.

\section{Conflicto de Intereses}

No existen conflictos personales, profesionales, financieroso de otro tipo.

\section{Consentimiento Informado}

Los autores cuentan con el consentimiento informado de los pacientes para la investigación, la publicación del caso y sus imágenes. 


\section{Referencias Bibliográficas}

1. Morillo M, Valencia V. Factores relacionados con la automedicación en los estudiantes de enfermería de la Facultad Ciencias de la Salud de la Universidad Técnica del Norte, en la ciudad de Ibarra en el periodo enero-agosto 2010. [Tesis de pregrado]; Ibarra: Universidad Técnica del Norte; 2010.29 p.

2. Moraga C, Padilla L. Factores asociados a la prevalencia de la automedicacion en los estudiantes usfx de chuquisaca en la ciudad de sucre gestion 2013. Archivos Bolivianos de Medicina. 2013; 20(88):19-25.

3. Chan M. Organización Mundial de la Salud. [Online]; 2015 [cited 2020 Julio 15]. Disponible en: https://www. who.int/dg/speeches/2015/traditional-medicine/es/.

4. Villegas F, Nasner K, Buitrago D, Cruz S, Ruden S, 17 . Bedoya $J$, et al. Patrón de automedicación en la zona urbana de la ciudad de Pereira (Colombia) en el trimestre marzo-mayo 2013. INVESTIGACIONES ANDINA. 2014; 29(6):1073-1085.

5. Gómez L, Galar M, Téllez A, Carmona F, Amaya A. Estudio de automedicación en una farmacia comunitaria de la ciudad de Toluca. Revista Mexicana de Ciencias Farmacéuticas. 2009; 40(1):5-11.

6. Caamaño A, Figueiras E, Lado J, J GO. La automedicación: concepto y perfil de sus «usuarios». Gaceta Sanitaria. 2000; 14(4):294-299.

7. Martín A, Alonso M, Iglesias de Sena H, Sáez M, Mirón J. Información sobre medicamentos y automedicación en las redes sociales. Rev. cuba. inf. cienc. salud. 2014; 25(2):145-156.

8. Salazar J, Bello L, Toledo A, Añez R, González L, Rojas $\mathrm{J}$, et al. Comportamiento epidemiológico de la automedicación y polifarmacia en individuos adultos del sector Dalia de Fernández, Municipio San Francisco. Archivos Venezolanos de Farmacología y Terapéutica. 2013; 32(1).

9. Barbero-González A, Pastor-Sánchez R, del Arco-Ortiz J, Eyaralar-Riera T, Espejo-Guerrero J. Demanda de medicamentos de prescripción sin receta médica. Atención Primaria. 2006; 37(2):78-87.

10. Cancho L, Echavarria G. Factores asociados a la automedicación en usuarios de la botica Biofarma de la ciuadad de Huancavelica - 2015 [Tesis de pregrado]. Perú: Universidad Nacional de Huancavelica; 2015. 125 p.

11. Ortiz F, Ortuño P, Ortega O. Estudio sobre automedicacion en poblacion mayor de 18 años del distrito de sarcobamba. Revista Científica Ciencia Médica. 2008; 11(1):5-9.

12. Sánchez F, Fornasini M. Determinación de las razones y diferencias en automedicación entre una parroquia urbana y una parroquia rural del Distrito Metropolitano de Quito [Tesid de pregrado]. Quito: Universdidad San Francisco de Quito; 2008.91 p.

13. Jara M, Jaramillo L, Macías J. Frecuencia de automedicación de AINES y analgésicos-antipiréticos y características que los rodean, en hogares de la parroquia San Blas de la ciudad de Cuenca en el año 2011 [Tesis de pregrado]. Cuenca: Universdiad de Cuenca; 2011. 77p.
14. Ministerio de Salud Pública. Ministerio de Salud Pública. [Online].; 2010 [cited 2020 Julio 15. Available from: https://www.salud.gob.ec/medidas-de-prevencion-contrala-influenza-lista-de-medicamentos-restringidos-deventa-libre/.

15. Ministerio de Salud Pública. Ministerio de Salud Pública. [Online].; 2012 [cited 2020 Julio 15. Available from: https://www.salud.gob.ec/ministerio-de-salud-publicafortalece-gestion-de-\%20farmacias-hospitalariasa-traves-de-la-implementacion-del-sistema-de$\% 20$ distribucion-de-medicamentos-positiva-por-dosisunitaria-sdmdu-en-\%20hospitales-del/.

6. Schwenkglenks M. Self-treatment and self-medication of Swiss primary care physicians: a cause for concern? Swiss Med Wkly. 2007; 137((7-8)).

7. Sánchez C, Nava M. Factores que infl uyen en la automedicación del personal de enfermería. Enf Neurol. 2012; 11(3):120-128.

18. Nogueira-Castillo M, Orejuela-Ramírez F, AndamayoFlores D. Factores asociados a la automedicación en pacientes que acuden a Servicios de Odontología de Hospitales del Perú. Rev Estomatol Herediana. 2018; 28(2):7277.

19. Salazar Z, Ochoa A, Arcos M, Guapisaca C, Rea D, Sánchez G. Factores asociados a la automedicación con antibióticos, Cuenca-Ecuador, periodo 2017. Archivos Venezolanos de Farmacología y Terapéutica. 2018; 1(2).

. Alcázar-Pichucho M, Zambrano-Santos R, Pincay-Pin V. Automedicación y los riesgos en la salud de la población adulta. Pol. Con. 2018; 3(8):434-448.

Recibido: 8 de octubre de 2020

Aceptado: 26 de octubre de 2020 
